\title{
High Resolution Observations of GRB 030329
}

\author{
Greg Taylor*, Dale Frail $^{*}$, Edo Berger ${ }^{\dagger}$ and Shri Kulkarni ${ }^{\dagger}$ \\ * National Radio Astronomy Observatory, Socorro, NM 87801, USA \\ ${ }^{\dagger}$ California Institute of Technology, Pasadena, CA 91125, USA
}

\begin{abstract}
The nearby ( $\mathrm{z}=0.1685)$ gamma-ray burst of 29 March 2003 has presented us with a unique opportunity to study an event with unprecedented physical resolution. This burst reached flux density levels at centimeter wavelengths more than 50 times brighter than any previously studied event. Here we present the results of VLBI observations that have resolved the radio afterglow, and constrain its proper motion in the sky to $<0.3$ mas. The size of the afterglow is measured to be $\sim 0.08$ mas 24 days after the burst, consistent with expectations of the standard fireball model. In observations taken 51 days after the burst we detect an additional compact, "jet", component at a distance from the main component of $0.28 \pm 0.05$ mas. The presence of this jet component is not consistent with the standard model.
\end{abstract}

\section{INTRODUCTION AND RESULTS}

GRB 030329, discovered by HETE-2 (GCN 1997), and localized rapidly in the optical bands (GCN 1985) represents a unique opportunity for VLBI observations. At a redshift of $z=0.1685$ (GCN 2020) this is the nearest cosmological burst detected to date. Observations with the VLA shows the radio afterglow to be the brightest detected so far, with a maximum flux of $55 \mathrm{mJy}$ at $43 \mathrm{GHz}$ one week after the burst [1].

The observations reported on here are based on the first five epochs taken between 3 and 83 days after the burst. All observations employed the VLBA ${ }^{1}$. Other telescopes used in one or more epochs were the Effelsberg 100-m telescope ${ }^{2}$ of the MPIfR, the phased VLA, the GBT, the Arecibo telescope (on June 19), and the WSRT tied array (on June 19). The observing runs were typically 5 hours long, with $256 \mathrm{Mbps}$ recording in full polarization with 2 bit sampling. The nearby $\left(1.5^{\circ}\right)$ source $\mathrm{J} 1051+2119$ was used for phase-referencing with a 2:1 minute cycle on source:calibrator. The weak calibrator $\mathrm{J} 1048+2115$ was observed hourly to check on the quality of the phase referencing.

In the first two epochs ( 3 and 8 days after the event) the GRB was observed at multiple frequencies within the 5 and $8 \mathrm{GHz}$ bands with the goal of studying the scintillation. The expected scintillation was found indicating a size $<0.05$ milliarcseconds (mas). A paper combining the flux density measurements from the VLBA and VLA in order to characterize the scintillation and contrain the source size at early times is in preparation.

The third and fourth epochs (at 24 and 51 days after the burst) were carried out at

\footnotetext{
1 The National Radio Astronomy Observatory is operated by Associated Universities, Inc., under cooperative agreement with the National Science Foundation.

2 The 100-m telescope at Effelsberg is operated by the Max-Planck-Institut für Radioastronomie in Bonn.
} 

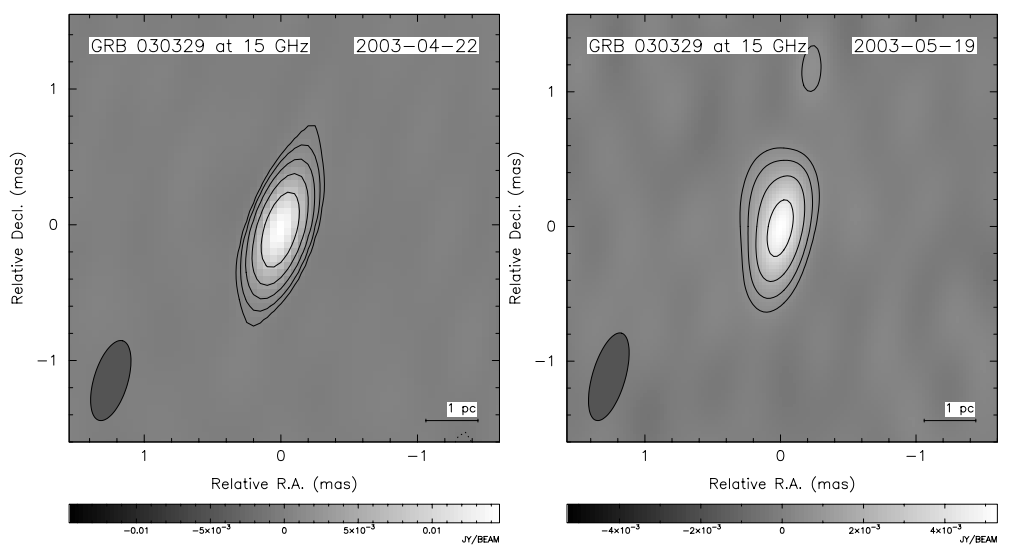

FIGURE 1. Images of GRB 030329 made with the VLBA and Effelsberg telescopes at $15 \mathrm{GHz}$ from 24 and 51 days after the burst respectively. During the May 19 epoch the GBT also participated. Contours are drawn starting at $0.5 \mathrm{mJy} / \mathrm{beam}$ and increase by factors of 2 . The beam pattern is shown in the lower left corner.

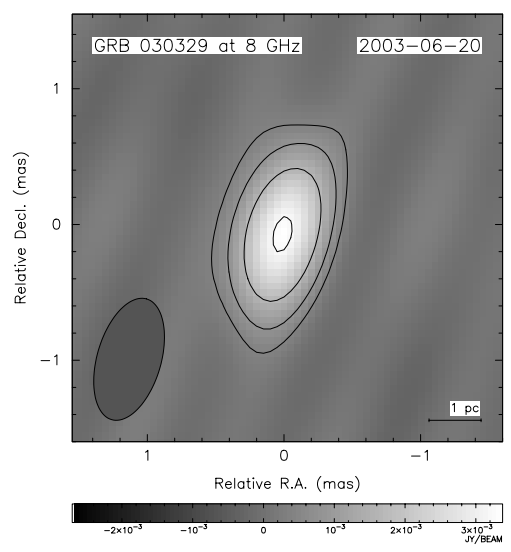

FIGURE 2. An image of GRB 030329 at $8.4 \mathrm{GHz}$ made with the VLBA, Effelsberg, Arecibo, phased VLA, and phased WSRT telescopes taken 83 days after the burst. Contours are drawn starting at 0.4 $\mathrm{mJy} /$ beam and increase by factors of 2 .

higher frequencies (15 and $22 \mathrm{GHz}$ ) in order to attempt to directly resolve the radio afterglow (see Fig. 1). The third epoch was best fit with a resolved Gaussian of size 0.08 mas, although a size of 0 mas cannot be completely ruled out. In the fourth epoch GRB 030329 appears resolved at $15 \mathrm{GHz}$ with a component appearing to the north east at $0.28 \pm 0.05$ mas from the main component. We tentatively identify this component as a fast jet component. To reach its position would require an average velocity of $19 \mathrm{c}$. This jet component is not detected in the $22 \mathrm{GHz}$ image, though this could be a result of the 


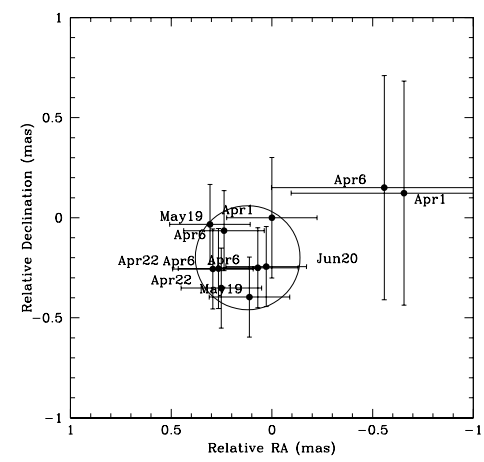

FIGURE 3. The positions derived from the observations to date relative to the first determination on April 1st. Observations at multiple frequencies at a given epoch have been plotted seperately since they are independent measurements. A circle with a radius of 0.3 mas is shown to encompass all current measurements at $8 \mathrm{GHz}$ and above.

reduced sensitivity at $22 \mathrm{GHz}$ and the faintness of the GRB. The reduction in SNR at 22 $\mathrm{GHz}$ makes removal of the residual atmospheric errors by self-calibration impossible.

The fifth epoch obtained 83 days after the burst, was carried out at $8.4 \mathrm{GHz}$ on June 19 using an impressive array consisting of the VLBA, GBT, phased VLA, phased WSRT, Effelsberg, and Arecibo. It was hoped that this epoch would confirm the existence of a jet component to the north east, but as shown in Fig. 2, there is only a slight indication of extended emission to the north east. The best fitting Gaussian model has a size of $0.17 \pm$ 0.04 mas. The synthesized beam of the uniformly weighted image shown in Fig. 2 has dimensions $0.92 \times 0.47$ mas.

The absolute positions derived from all the epochs can be combined in order to constrain the proper motion of the burst. The positions for each frequency and epoch are plotted in Fig. 3.

\section{IMPLICATIONS FOR THE MODELS}

The "cannonball" model $[4,3]$ predicts a motion of 0.05 mas/day, or $\sim 1$ mas between 3 and 24 days after the burst. This motion is clearly excluded by our observations (see Fig. 3), and between 3 and 83 days after the burst the motion is less than 0.004 mas/day $(<14 \mathrm{c})$. At the redshift of 0.1685 , a motion of 1 mas/year corresponds to $10.9 \mathrm{c}$. Based on these observations we can rule out the fast cannonball model as currently proposed.

Fireball models of heating by a single relativistic shock front predict that at late times the fireball should look like a ring [5]. Modeling of afterglow light curves in the optical and radio suggest that they are not isotropic events, but are jetted with an initial opening angle of $\theta_{0} \sim 0.1 \mathrm{rad}$. The size of the fireball depends on the observer's viewing angle, but presumably observers at large angles to the jet could not have seen the burst of gamma-rays. [6] predict an angular size for GRB 030329 that grows linearly with time up to a size of $\sim 0.27\left(E_{51} / n_{0}\right)^{1 / 3}$ mas some 100 days after the burst and a somewhat 
slower $\left(t^{0.4}\right)$ growth after that. VLBI observations at $22 \mathrm{GHz}$ show that GRB 030329 had a size of $\sim 0.08$ mas just 24 days after the burst, roughly consistent with the energies $\left(E_{51}\right)$ and exterrnal densities $\left(n_{0}\right)$ derived by [2], but it is not possible to discriminate between a disk and a ring. [6] also predict a shift in the flux centroid from early times by 0.019 mas, but this is well below our current capabilities to measure. In addition to the predictions of the fireball model, in GRB 030329 an extension was detected 50 days after the burst at 0.28 mas from the main component (Fig. 1). This is the first direct evidence for a jet in GRBs, and may indicate that some very fast shocks are produced, though none have been predicted by the standard fireball model.

\section{FUTURE PLANS}

Although GRB 030329 has faded considerably, it is still detectable with VLBI techniques. One year after the burst the diameter of the ring is predicted by the standard fireball model to be $3 \times 10^{18} \mathrm{~cm}$ or 0.33 mas. This size can be resolved by VLBI observations at $8 \mathrm{GHz}$. A further epoch is planned for the Global VLBI session in Fall 2003, and another epoch beyond that may be possible if the sensitivity can be improved by an increase in bandwidth.

\section{ACKNOWLEDGMENTS}

We are particularly greatful to the schedulers of the VLBA, GBT, Effelsberg, WSRT, and Arecibo telescopes for heroic efforts on behalf of this program.

\section{REFERENCES}

1. Berger, E. et al. ApJ 560, 652 (2001).

2. Berger, E. et al. Nature 426, 154 (2003).

3. Dado, S., Dar, A., \& De Rujula, A., A\&A 401, 243 (2003).

4. Dar, A. et al., GCN 2133 (2003).

5. Granot, J., Piran, T., \& Sari, R., ApJ 513, 679 (1999).

6. Granot, J., \& Loeb, A., ApJ 593, L81 (2003). 